\title{
EFSA Scientific Network of Risk Assessment of Nanotechnologies in Food and Feed Annual Report 2014
}

(C) Springer - CEC Editore 2015

The Annual Report of the EFSA Scientific Network of Risk Assessment of Nanotechnologies in Food and Feed for 2014 has recently been published [1]. It is also available online: www.efsa.europa.eu/ publications. In accordance with the EFSA's strategy for cooperation and networking with Member States, a Network for Risk Assessment of Nanotechnologies in Food and Feed was established in 2010. The Nano Network had its inaugural meeting in February 2011 and following this, one meeting per year is scheduled. The overall goals of this Network are to facilitate harmonisation of assessment practices and methodologies; to enhance exchange of information and data between the EFSA and Member States; and to achieve synergies in risk assessment activities. The annual reports of the Network inform the public and the EFSA Advisory Forum about its specific activities and achievements. During 2014, the Network followed up on its priority areas and contributed to the creation of inventory lists of applications of nanomaterials already present in the food/feed chain. During its meeting in 2014, the Network dedicated most of its discussions to relevant research results on possible toxic effects following the oral route of exposure. The Network exchanged views on the technical aspects and implications of the definition for Nanomaterial. The network also shared its views on the ongoing and upcoming risk assessments of the EFSA on applications implicitly or explicitly comprising nanoforms. The Network updated its list with national research and contact details of national laboratories that can analyse nanomaterials in complex matrices. The Network is composed of representatives from 21 Member States and Norway. In addition, observers of this Network represent the Former Yugoslav Republic of Macedonia, Turkey and Montenegro. There is also representation from the European Commission (DGSANTE and JRC), the EFSA Scientific Committee and the relevant Units/Panels.

\section{Summary of the $\mathbf{2 0 1 4}$ report}

During 2014, the Network followed up on its priority areas and contributed to the creation of inventory lists of applications of nanomaterials already present in the food/feed chain. At its 2014 meeting, the Network focussed again on updates of research results from toxicological studies relevant to the oral route of exposure. Member State representatives presented relevant studies.

\section{EFSA}

Parma, Italy

scientific.committee@efsa.europa.eu
The type of nanomaterials that are now occurring in the food/feed chain are mainly titanium dioxide $\left(\mathrm{TiO}_{2}\right)$ and synthetic amorphous silica (SAS). The evidence bases for oral toxicity and for conducting comprehensive risk assessments of these two materials is building up, but more research is needed. Challenges to making firm risk assessment conclusions are as follows: (i) the intake estimation, (ii) the possible worst-case absorption and the dose dependence of absorption, (iii) the potential irrelevance of high-dose oral toxicity studies for risk assessment, (iv) the extrapolation of kinetic data from rats to humans, (v) the nanoparticle determination in tissues and (vi) the many differences between the types of nanoforms of one nanomaterial (e.g., in kinetics and toxicity). Some differences in behaviour of different nanoforms have been observed, but there is no clear overview. A new issue of concern is that absorption is not linear with dose: high-dose studies are often used for toxicity testing for estimation of safe dose, while the high dose may result in aggregation, agglomeration, gelation and, as a consequence, dosedependent absorption. Challenges also remain regarding the technical aspects of considering a material as a nanomaterial (NM) for the regulatory purpose of food labelling. By 2017, the NanoDefine project (FP7) is expected to deliver an implementable test scheme for regulatory purposes to distinguish nano from non-nano. The Network agreed that regardless of the current challenges and regardless of the percentage of nanoforms in the bulk material (particle size percentage or mass percentage), the EFSA should assess the nano-fraction, no matter how small. Food law, as implemented by the EFSA Panels, covers nanomaterials. Nanomaterials are addressed mainly by cross-referring to the "Guidance on the risk assessment of the application of nanoscience and nanotechnologies in the food and feed chain" [2]. The Network also updated its list with contact details of national laboratories that have equipment and expertise for analysing certain nanomaterials in complex matrices.

\section{References}

1. European Food Safety Authority. 2015. Annual report of the EFSA Scientific Network of Risk Assessment of Nanotechnologies in Food and Feed for 2014. EFSA supporting publication 2014: EN-762. $11 \mathrm{pp}$

2. EFSA Scientific Committee. 2011. Guidance on the risk assessment of the application of nanoscience and nanotechnologies in the food and feed chain. EFSA J 9:2140. http://www.efsa.europa.eu/en/efsajournal/doc/2140.pdf 